\title{
Aplicación difusora de información para dispositivos Android OS en Ciudad Universitaria, UNAH
}

Irma Gámez Suazo

\section{RESUMEN}

El estado actual de las tecnologías de la información y comunicación permite que instituciones de educación superior cuenten con canales virtuales de comunicación para difundir información institucional. La dependencia de documentación escrita en la institución, las limitaciones que eso conlleva y la falta de un canal virtual de comunicación único en el cual se publique información oficial, hace que en muchas ocasiones los estudiantes no estén informados adecuadamente sobre lo que está ocurriendo en su respectiva carrera. Así, el objetivo de este trabajo es evaluar un prototipo de aplicación de una difusora de información universitaria. Se eligió como unidad de análisis a los estudiantes de la Carrera de Informática Administrativa, ya que las instalaciones del Departamento cuentan con un centro de datos apto para facilitar el acceso a la aplicación a través de conexión WiFi. El prototipo se puso a disposición de la comunidad universitaria correspondiente a la matrícula del segundo período del año 2015 en Ciudad Universitaria. Después de utilizarlo, se les pidió que completaran un cuestionario virtual para que expresaran su opinión sobre el funcionamiento de la aplicación. Para los estudiantes, la comunicación de noticias oficiales constituye una guía para mejorar su conocimiento sobre novedades en su Carrera, de forma que la aplicación ayuda a que las unidades académicas logren difundir información de forma eficaz, permite llevar un registro estadístico de noticias difundidas y reduce el gasto de papelería. Los resultados de las pruebas realizadas mostraron que la aplicación es capaz de informar efectivamente a la comunidad universitaria.

Palabras clave: Android, servicio web, noticias.

\footnotetext{
${ }^{1}$ Beneficiaria de una beca básica de la DICYP, profesora del Departamento de Informática Administrativa, Facultad de Ciencias Económicas, UNAH: irma.gamez@unah.edu.hn
} 


\section{ABSTRACT}

The status of the Information Technology and Communication allows existing institutions, both public and private higher education have virtual communication channels to disseminate institutional information. Students, either distance learning or face, make use of social networks and websites where authorities of their respective academic departments give out news about the career. The dependence of documentation written in the institution, the limitations that entails, and the lack of a single virtual communication channel where official information is published, makes that often students are not adequately informed about what is happening in the University and within their respective career. The aim of this paper is to analyze the requirements and architecture of a diffuser application information, to carry out the development of a functional prototype and validate the results of the implementation of that prototype. In this project a quantitative methodology was applied, with non-experimental cross-sectional design. Students of the Administrative Computing career were chosen as the object of study, since the facilities of the Department's data center offers a secure installation of telecommunications equipment and thus, facilitate access to the application through WiFi connection. The prototype was made available to the community enrolled during the first term of 2015 in Ciudad Universitaria and was asked to complete a questionnaire to express their opinion. For students, the official news media is a guide to enhance their knowledge of novelties in his career. The application helps to achieve the academic units disseminate information effectively, efficiently, can keep a statistical record of broadcast news and reduces the cost of stationery. The results of tests showed that the application is capable of effectively inform the university community.

Keywords: Android, web service, news. 


\section{INTRODUCCIÓN}

El estado actual de las tecnologías de la información y comunicación permite que instituciones de educación superior, públicas y privadas, cuenten con canales virtuales de comunicación para difundir información institucional. Los estudiantes en modalidad a distancia o presencial, tienen a su disposición redes sociales y sitios web en donde las autoridades de sus respectivos departamentos académicos les dan a conocer noticias sobre la carrera. La dependencia de documentación escrita en la institución, las limitaciones que eso conlleva y la falta de un canal virtual de comunicación único en el cual se publique información oficial, hace que en muchas ocasiones los estudiantes no estén informados adecuadamente sobre lo que está ocurriendo en su centro universitario y dentro de su respectiva carrera. Asimismo, los departamentos académicos podrían ahorrar en gastos de papelería al disminuir la cantidad de documentos impresos.

Desde su aparición, los teléfonos móviles han estado en constante evolución, pasando desde un teléfono básico utilizado para realizar llamadas y envío de mensajes SMS, hasta un dispositivo sofisticado capaz de ejecutar programas desarrollados por terceros, descargados desde plataformas como App Store de Apple y Play Store de Google. Una aplicación para dispositivos móviles es un vehículo idóneo para proporcionar a los estudiantes la información que necesitan, ya que es bien conocida la popularidad de estos dispositivos en la sociedad del conocimiento en la que vivimos. A través de la aplicación puede mostrarse al estudiante las noticias sobre su carrera y de otros departamentos académicos de su interés.

El propósito de este artículo es evaluar un prototipo de aplicación de una difusora de información universitaria. Para lograr satisfactoriamente este objetivo, el proyecto necesita un sitio web desde donde la aplicación obtendrá la información y la dirigirá a los clientes móviles. Así, el Departamento de Informática Administrativa está anuente a proporcionar los insumos necesarios que estén disponibles para el desarrollo del proyecto. No se tomarán en cuenta dispositivos con otros sistemas operativos como iOS, Blackberry o Windows Phone.

Para los estudiantes, la comunicación de noticias oficiales constituye una guía para mejorar su conocimiento sobre novedades en su carrera. La aplicación también ayuda a que las unidades académicas logren difundir información de forma rápida, eficaz y eficiente; lo cual podría permitir llevar un registro estadístico de noticias difundidas y reducir el gasto de papelería. 
La revisión de la literatura muestra que la comunidad científica ha puesto mucho esfuerzo en la investigación y exploración de los beneficios de la difusión de información a través de medios electrónicos. Existen varios aportes de otros investigadores que ayudan a problematizar la investigación. El desarrollo del prototipo está en parte influenciado por el artículo de Sajjanhar y Zhao (2012), quienes proponen un método para ofrecer noticias filtradas de canales RSS a un cliente móvil, utilizando servicios web de geocodificación para implantar el acceso basado en la localización del sitio web emisor de la información.

Desde el punto de vista metodológico, no se tomará en cuenta la geolocalización, ya que no es un elemento primario en el filtro de noticias del campus, aunque posiblemente sea útil en una futura versión que se aplique en todos los centros regionales de la institución.

La aplicación también es novedosa en la UNAH, ya que actualmente no existen proyectos en ejecución, ni en desarrollo, conocidos que incluyan aplicaciones difusoras de información. Muchas universidades extranjeras han desarrollado aplicaciones móviles para mejorar la interacción con sus estudiantes, empleados y visitantes. Ofrecen todas las funcionalidades de una red social como servicios de mensajería, publicación de videos, mapa del campus, noticias, entre otros; por ejemplo, la Universidad de Hardvard (Harvard University, 2014) y la Universidad Politécnica de Valencia (UPV, 2014). Otras se valen de la aplicación Google Maps para ubicar al estudiante en el campus e informarle sobre el entorno académico (UNAM Mobile, 2014). En este proyecto, obtener un mapa georreferenciado de la ciudad universitaria o desarrollar una red social no es alcanzable en este proyecto, ya que se necesita información específica de toda la comunidad universitaria y un mayor tiempo de desarrollo. La utilización de sistemas operativos menos populares es un trabajo futuro que está disponible en nuevas versiones de la aplicación.

Los servicios web son módulos de computación reutilizables que proveen una funcionalidad predefinida (W3C, 2004). Estos habilitan una nueva clase de aplicaciones llamadas basadas en servicios, que delegan su flujo de trabajo a servicios externos. El resto del artículo se organiza de la siguiente forma: la sección I presenta una descripción general del método utilizado que incluye el diseño del experimento, población donde se validó el prototipo de la aplicación y la descripción del sistema desarrollado. En la sección II se analizan y discuten los datos obtenidos mediante la validación del prototipo. Por último, la sección III presenta las conclusiones y el trabajo futuro. 


\section{METODOLOGÍA}

En este apartado se detalla la fundamentación metodológica que incluye el tipo de diseño de investigación, la población del estudio, una descripción del sistema y, por último, el análisis estadístico de los datos obtenidos de los instrumentos diseñados para este proyecto. A continuación se describe cada uno de estos elementos.

\section{Diseño del experimento}

El diseño que se ha adoptado en el proyecto es el no experimental transversal exploratorio (Hernández, Fernández y Baptista, 2010), ya que se recolectaron datos en un momento específico proporcionado por un grupo definido, sin intervención en el ambiente. El prototipo de la aplicación se puso a disposición de estudiantes, docentes y personal administrativo de la Carrera de Informática Administrativa, correspondiente al segundo período del año 2015 en ciudad universitaria, a través de la dirección electrónica http://200.106.236.141/informativa/index.php.

Después de usar el prototipo, se pidió a los usuarios que completen un cuestionario para expresar su opinión sobre el funcionamiento de la aplicación. Se prepararon dos cuestionarios, uno para aplicarse a los usuarios (ver anexo 1) y otro para los administradores del portal web (ver anexo 2). El cuestionario para estudiantes se divide en las dos dimensiones organizadas en el apartado Operacionalización de variables, que incluye cinco preguntas cerradas con escala de opinión tipo Likert ${ }^{2}$ (1: Totalmente en desacuerdo, 2: En desacuerdo, 3: Neutro, 4: De acuerdo, 5: Totalmente de acuerdo). Ambos cuestionarios consideran la variable Validación de la aplicación en las dimensiones Funcionalidad y aceptación, para medir el grado de cumplimiento de las funcionalidades que la aplicación ofrece a los usuarios.

El cuestionario dirigido a los estudiantes recolectó su opinión con respecto a la aceptación del prototipo. El cuestionario dirigido a los administradores del portal web se organizó en tres partes. La primera recolectó la opinión sobre la funcionalidad y la segunda parte la aceptación de la aplicación; ambas partes contienen preguntas cerradas con escala de opinión tipo Likert, además de preguntas abiertas. La tercera parte contiene tres preguntas abiertas y se ocupa de obtener sugerencias y recomendaciones que se pudieran aplicar a futuras versiones del prototipo.

\footnotetext{
${ }^{2}$ Escala psicométrica comúnmente utilizada en cuestionarios.
} 


\section{Población del estudio}

En el Departamento de Informática Administrativa existen 2,500 estudiantes matriculados, que representan la población. ${ }^{3}$ Calculando el tamaño de la muestra con un nivel de confianza del $95 \%$ e intervalo de confianza, se obtiene una muestra de 748 estudiantes. La selección de esta cantidad de estudiantes se considerará al azar. Además, se contará con un cuestionario aplicado a los administradores del portal web de la carrera.

\section{Descripción general del sistema}

Una vez detallada la fundamentación metodológica y la población de estudio, se describe la forma en que se orienta la solución al problema que se plantea. La figura 1 ilustra el funcionamiento general del sistema.

\section{Figura 1. Diagrama conceptual de la solución}

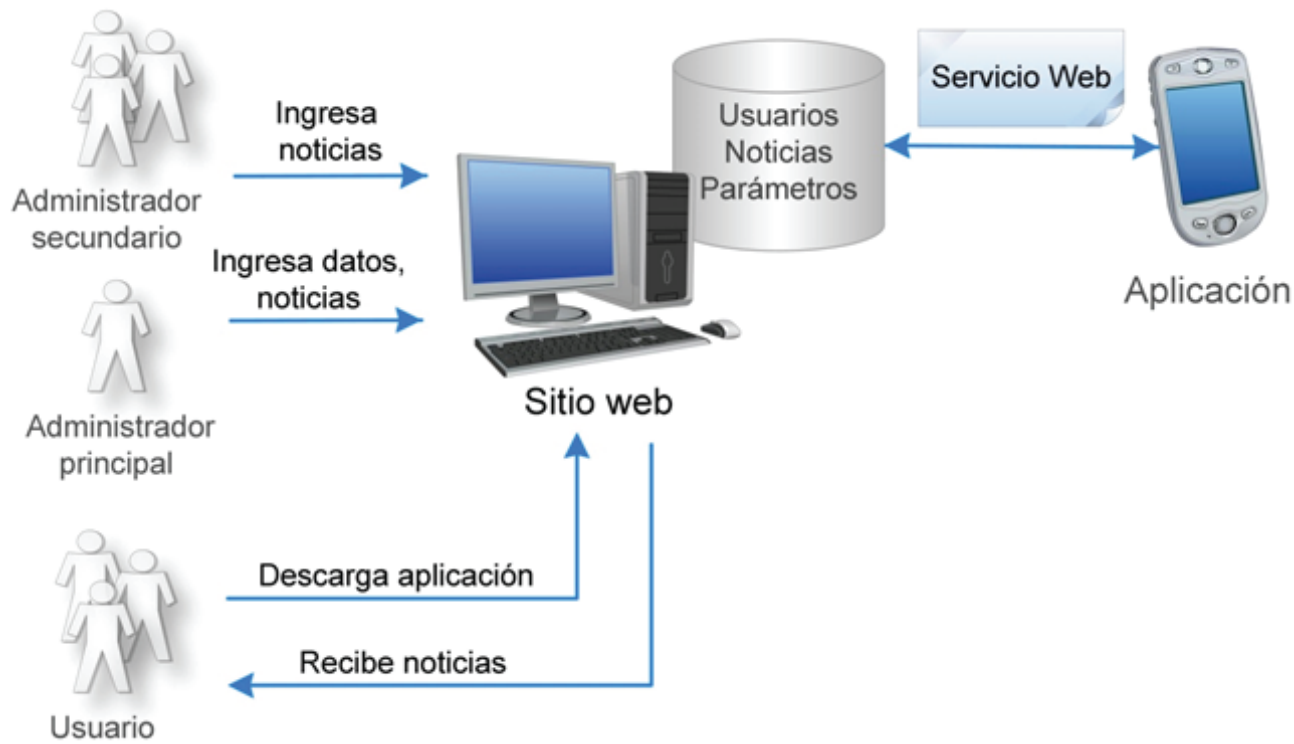

${ }^{3}$ Dato de matrícula obtenido de la Jefatura del Departamento de Informática Administrativa para el segundo período de 2015. 
En el sistema se identifican tres tipos de usuarios: el primero es el administrador principal, que es único y goza de acceso ilimitado a todas las funciones del sistema; el segundo es el administrador secundario, con acceso limitado a tales funciones y pueden existir cuantos se necesiten en la unidad académica; por último el usuario, que es la persona que utiliza la aplicación en su dispositivo móvil.

Los administradores ingresan al sitio web con un usuario y contraseña previamente definidos, visualizan un formulario en el que ingresarán los datos de la noticia, además de una serie de opciones con las que pueden administrar los usuarios de la aplicación y comunicarse con ellos mediante mensajería. El usuario ingresa al sitio web desde su dispositivo móvil, visualiza un formulario del cual elegirá el centro, facultad y departamentos de los que desea recibir noticias. Luego, debe registrarse para descargar la aplicación. Posteriormente recibirá un correo electrónico confirmando su solicitud de registro y un enlace para descargar la aplicación.

Una vez instalada, la aplicación comenzará a buscar las noticias que correspondan a las preferencias que el usuario eligió al momento de registrarse y lo alertará mediante notificaciones, estas constituyen un acceso directo a la aplicación en donde se encuentra el cuerpo de la noticia, la lista de noticias vigentes y la configuración de las preferencias. La siguiente lista de requerimientos define el comportamiento general del sistema y expone las principales funciones que el prototipo ofrece a los usuarios:

1. Generación de tabla de preferencias del usuario. El usuario puede elegir más de un centro, una facultad y un departamento del que desea recibir noticias.

2. Desarrollo de un sitio web desde donde se administren las noticias.

3. Desarrollo del mecanismo encargado de emitir las notificaciones, este constituye el elemento que se ejecuta en el dispositivo móvil del usuario y que se administra desde el sitio web mediante servicio web.

Según los requerimientos analizados, la emisión de notificaciones implica filtrar las noticias que correspondan a las preferencias de determinado usuario en su respectivo dispositivo móvil. A continuación se describe el diseño conceptual de la solución al problema que se plantea:

1. Desarrollo del prototipo: el diseño de la arquitectura general de la aplicación está construido en tres capas: presentación, negocios y datos. La decisión de optar por un diseño en capas es principalmente para descomponer la aplicación en subtareas. En la capa de negocios está el valor real del sistema. Se divide en los módulos: administrador de preferencias, administrador de noticias y servicio. 
2. Estructura de la capa de negocios: el diagrama que se muestran en la figura 2 ilustra las clases utilizadas para encapsular las funcionalidades de los módulos.

Figura 2. Diagrama de clases de la capa de negocios

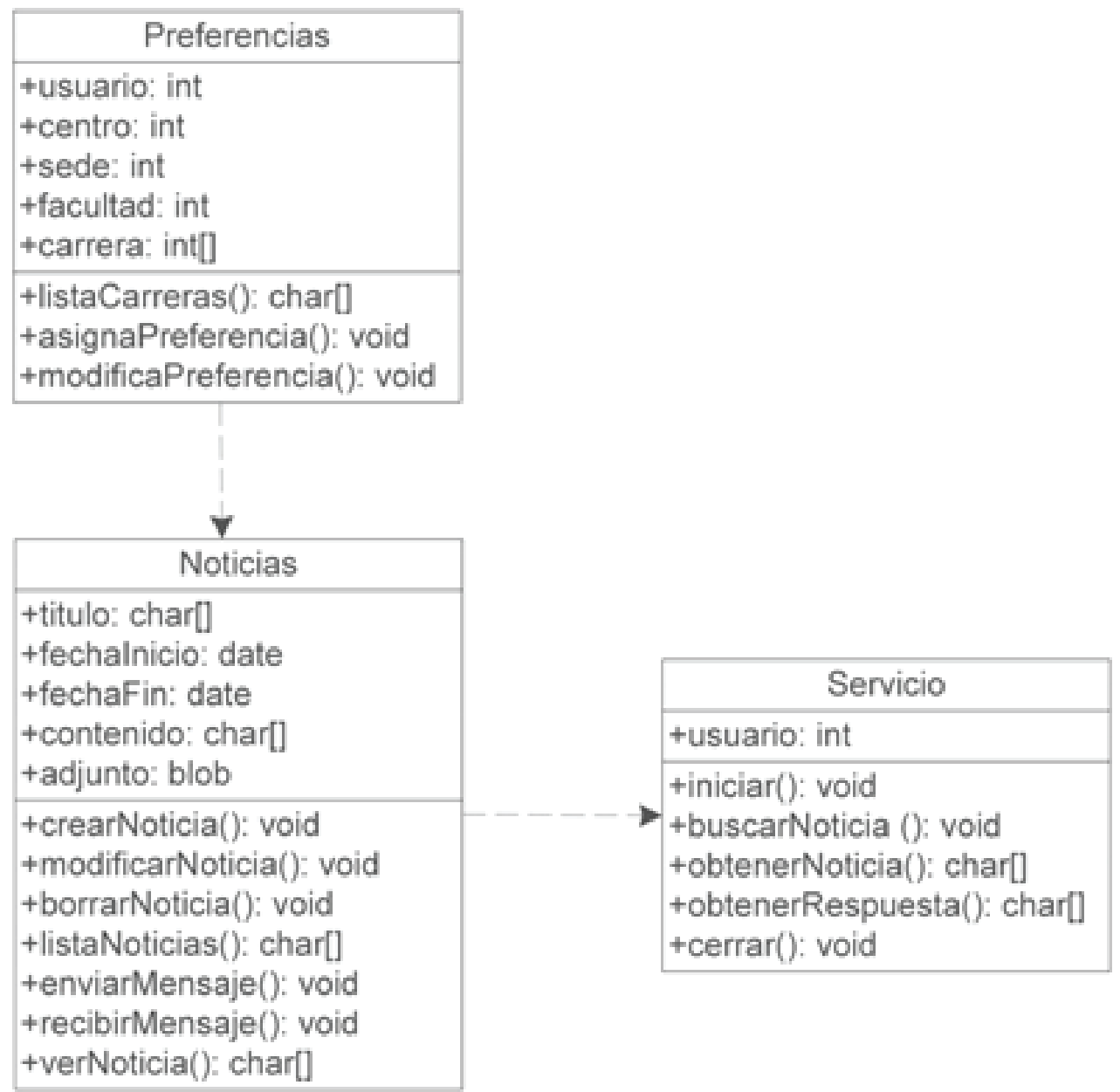

Fuente: propia 
Cada una de las clases se detalla a continuación:

a. Administrador de preferencias: es el encargado de recolectar y validar los datos de entrada del usuario. El módulo alimenta una tabla en la base de datos que servirá como insumo al administrador de noticias para que el usuario reciba solo información emitida por los departamentos académicos que haya seleccionado.

b. Administrador de noticias: en este componente es donde se obtienen las noticias que se mostrarán al usuario, después de ejecutar un hilo en segundo plano para conectarse con el servidor web. Básicamente implementa cuatro métodos: el primero es el encargado de manipular la creación, modificación y borrado de noticias en el sitio web; el método ver noticia permite leer las noticias vigentes en la barra de notificaciones y habilita el acceso al envío de mensajes al administrador; lista noticias muestra al usuario la lista de noticias previamente recibidas en su dispositivo; por último, enviar mensaje y recibir mensaje se utilizan para el servicio de mensajería entre los usuarios y el administrador del sitio web.

c. Servicio: encapsula las funciones destinadas a establecer la conexión entre la aplicación y la página web mediante servicio web. La comunicación se establece con el método iniciar, que se ejecuta al lanzar la aplicación y se mantiene activo como servicio hasta que sea cerrado por el usuario. El método buscar noticia muestra las noticias y respuestas para el usuario en la barra de notificaciones, después de verificar en las preferencias del usuario si se encuentra seleccionada la carrera de la cual proviene la noticia. La recepción de mensajes por parte del administrador se gestiona con el método obtener mensaje. Una vez que el usuario cierra la aplicación, se ejecuta el método cerrar para detener el servicio.

3. Implementación. Las aplicaciones que se desarrollan para el sistema operativo Android se escriben en el lenguaje de programación Java; por esta razón, el prototipo se desarrolló en tal lenguaje. En el contexto de Android, una aplicación se conoce como paquete y consta de una colección de código Java y tablas de base de datos. Para manejo de la base de datos, se utilizó MySQL. La conexión a base de datos es transparente gracias al modelo unificado de datos de Java. En cuanto al desarrollo del sitio web, se utilizó el lenguaje PHP y Javascript.

4. Pruebas y validación. Antes de poner el prototipo a disposición de los usuarios se realizaron muchas pruebas que se concentraron en verificar el funcionamiento correcto de las acciones visibles para el usuario y las salidas del sistema. Los datos que se utilizaron en las pruebas fueron proporcionados por 748 estudiantes y docentes de la Carrera de Informática Administrativa. Las pruebas abarcaron la creación de noticias, creación de usuarios, envío y recepción de mensajes 
desde el lado de la página web, así como la elección de preferencias, lectura de noticias y mensajería del lado de la aplicación.

Los resultados obtenidos mostraron que el servicio web funciona muy bien como mecanismo de conexión aplicación-página web. Hace posible que el cliente reciba las noticias en forma de notificaciones sin la necesidad de lanzar la aplicación. A continuación se detalla el proceso para validar el prototipo en un entorno real con la intervención de los usuarios finales. Se instaló una versión operacional en un servidor privado. Se invitó a los estudiantes, administradores y docentes a que ingresaran a la dirección electrónica http://200.106.236.141/informativa/index.php. Los estudiantes se autenticaron con su número de cuenta y el personal con su número de empleado. Es importante destacar que solo pueden descargar la aplicación los estudiantes matriculados en la UNAH y los usuarios que la deseen con previa aprobación del administrador principal.

El prototipo estuvo disponible del 9 de septiembre al 25 de septiembre de 2015. Después de usar el prototipo se pidió a los usuarios que completaran un cuestionario para expresar su experiencia; para esto se prepararon dos cuestionarios, uno para aplicarse a los clientes, que incluyen estudiantes y docentes; el otro para el grupo de administradores. Ambos cuestionarios consideraron la variable Validación de la aplicación en las dimensiones Funcionalidad y aceptación, para medir el grado de cumplimiento de las funcionalidades que la aplicación ofrece a los usuarios. La siguiente sección detalla las opiniones de los usuarios después de interactuar con el prototipo.

\section{ANÁLISIS Y DISCUSIÓN DE DATOS}

Opinión de los usuarios: con el cuestionario dirigido a los estudiantes se recolectó su punto de vista con respecto a la utilidad de tener acceso a las noticias relacionadas con otras carreras, siendo este uno de los requerimientos identificados en la etapa de análisis. La figura 3 muestra la distribución de frecuencias que ilustra las respuestas de los estudiantes. Aquí puede apreciarse que predomina el interés en conocer la actualidad de otras carreras, debido a que todos los planes de estudio de las carreras de la UNAH incluyen asignaturas impartidas por distintas unidades académicas. Aunque no se identifica una diferencia clara entre las distintas alternativas, es importante notar que en la mayoría de los encuestados se observa la necesidad de filtrar el contenido que se entrega a través de los canales virtuales de comunicación. 
Figura 3. Utilidad de noticias relacionadas con otras carreras

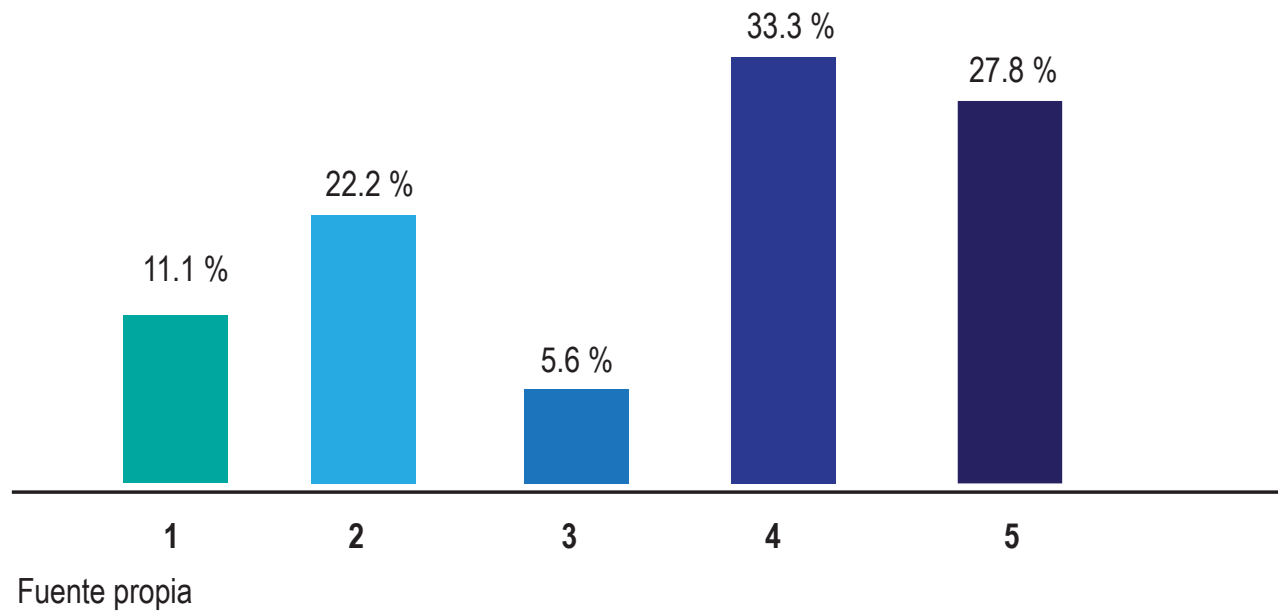

Se preguntó explícitamente a los usuarios si consideran que aplicación puede ayudarles a mantenerse informados sobre la actualidad de su unidad académica. En la figura 4 se aprecia que el $83.3 \%$ de ellos está de acuerdo con que la aplicación es capaz de proporcionarles un medio accesible de difusión de noticias.

Figura 4. Utilidad de la aplicación

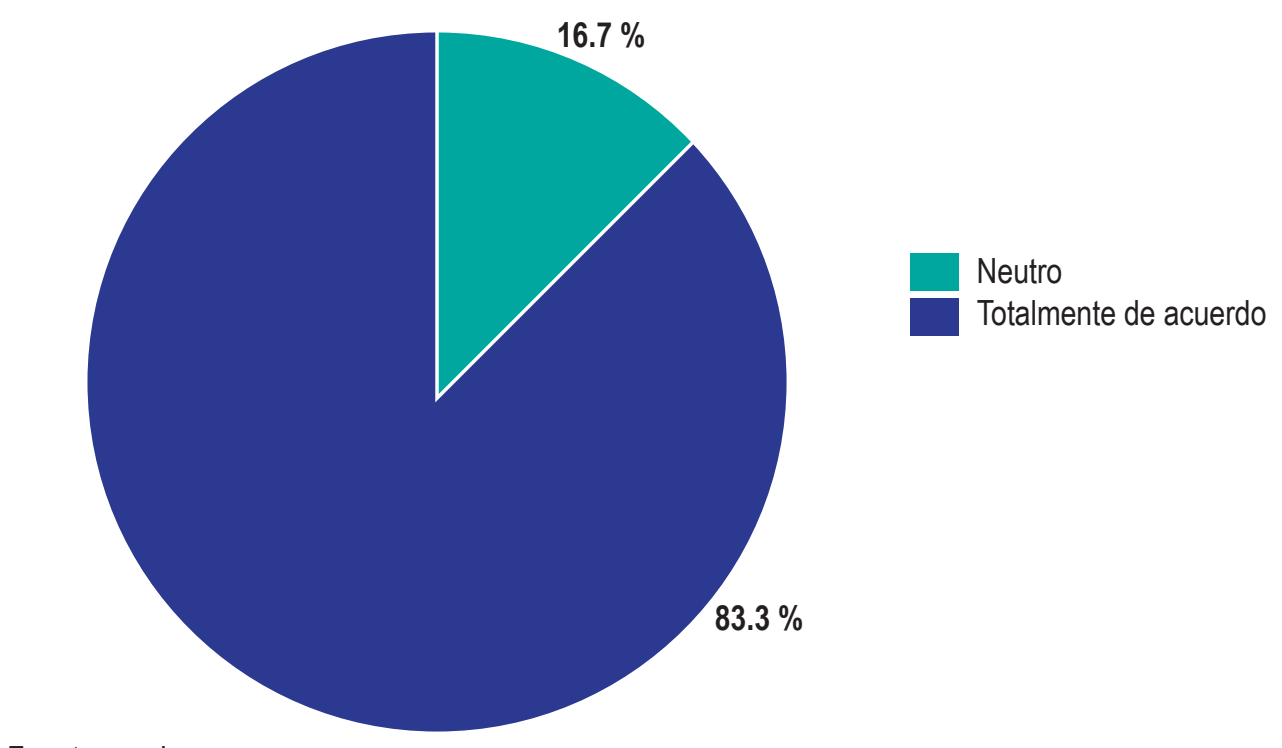

Fuente propia 
1. Opinión de los administradores: la variable que se considera en el cuestionario es validación de la aplicación en la dimensión funcionalidad. Los resultados mostraron que el grupo focal está de acuerdo con que la aplicación puede ayudar a mantener informada a la comunidad estudiantil y al personal docente sobre la actualidad en el Departamento de Informática Administrativa, siempre que se garantice que la aplicación pueda vincularse con los espacios de publicación de noticias que ya existen: Facebook y la página oficial de la UNAH.

Las recomendaciones implican que el sistema puede tener varias mejoras. Una de ellas es la posibilidad de marcar como leída una noticia para evitar que el usuario vuelva a recibirla como reciente. Otra recomendación es diseñar métodos para construir registros históricos de visitas a noticias (estudios con información histórica estaban fuera del alcance del trabajo en su etapa inicial). En general, los resultados de las pruebas y la validación demuestran que la arquitectura del prototipo es válida y resuelve el problema planteado. Los resultados también indican varias mejoras para desarrollos futuros del sistema. Las posibles mejoras se discuten en la siguiente sección.

\section{CONCLUSIONES}

Este artículo describe la metodología de desarrollo de un sistema para difundir noticias filtradas según el departamento emisor de la noticia que utiliza servicio web como mecanismo de envío de notificaciones a un dispositivo móvil y la subsecuente implementación del prototipo. El prototipo fue evaluado exitosamente con usuarios reales y los resultados obtenidos son prometedores. Enseguida se exponen las conclusiones obtenidas.

a. Los resultados obtenidos en la etapa de pruebas y validación indican que el enfoque propuesto es capaz de ayudar a mantener informada a la comunidad estudiantil sobre la actualidad en el Departamento de Informática Administrativa y, por lo tanto, su beneficio se generaliza a las demás unidades académicas.

b. Las contribuciones más novedosas de este trabajo son: una propuesta de un boletín universitario personalizado que alerte al usuario cada vez que las unidades académicas de su interés emitan una noticia, ofrecer a las autoridades una herramienta unificada que les informe qué está ocurriendo en las unidades académicas y la utilización de servicios web como mecanismo de comunicación de noticias. 
c. El diseño de la solución propuesta fue validado por usuarios reales y expertos que interactuaron con el prototipo a través del sitio web. Los resultados mostraron que los usuarios están de acuerdo con que la aplicación es un medio de información útil para la comunidad universitaria.

\section{AGRADECIMIENTO}

A la jefe del Departamento de Informática Administrativa, Sandra Lorena Velásquez, por su anuencia en el desarrollo de este proyecto. Asimismo, a la Dirección de Investigación Científica y Posgrado de la UNAH, por la oportunidad de participar y fortalecer el área de investigación en la carrera.

\section{BIBLIOGRAFÍA}

Android Developers. (2014). Android Studio. Obtenido de: http://developer.android.com/sdk/index.html

Android SDK. (2014). Get the Android SDK. Obtenido de: http://developer.android.com/sdk/index.html

Harvard University . (2014). All Harvard. Obtenido de: http://www.harvard.edu/all-harvard-mobile

Hernández, R. Fernández, C. y Baptista, P. (2010). Metodología de la investigación. México: McGraw Hill.

Sajjanhar, A., \& Zhao, Y. (2012, September). Web service to deliver filtered RSS items to a mobile application. En 2012 Seventh ChinaGrid Annual Conference (pp. 128-133). IEEE.

UNAM Mobile. (2014). UNAM 360. Obtenido de: http://unam.mobi/en/projects/unam-360

UPV. (2014). Aplicación UPV para móviles inteligentes. Obtenido de: https://www.upv.es/apps/index-es.html

W3C. (2004). Web Services Architecture. Obtenido de: http://www.w3.org/TR/ws-arch 